\title{
Localization through compressive sensing: A survey
}

\author{
A. Ali \\ TRENDS Lab, ITU University, Lahore, Pakistan \\ Email address: \\ anum.ali@live.com
}

\section{To cite this article:}

A. Ali. Localization through Compressive Sensing: A Survey. International Journal of Wireless Communications and Mobile Computing. Special Issue: Localization by Compressive Sensing. Vol. 3, No. 2-1, 2015, pp. 1-5. doi: 10.11648/j.wcmc.s.2015030201.11

\begin{abstract}
User mobile device or for wireless node detection localization is a primary concern not only in normal days but especially during emergency situations. There is variety of useful and necessary applications related to localization and it is an important technology playing critical role in wireless communication. The conceptual point of view is to sense the localization (coordinates of the user) from a specific region of interest (ROI). For reducing the complexity and increasing efficiency, the data samples for location sensing is limited in a term of taking sparsity of the detected signal in known transformed domain by taking fewer data samples. This whole phenomenon is called compressive sensing. This paper introduces this technology especially in location-sensing and discusses the present techniques.
\end{abstract}

Keywords: Cognitive Radio, Localization, Mobile Networks, Wireless Networks, Sparsity, Compressive Sensing, Signal Detection

\section{Introduction}

In mobile and wireless network architecture location and mobility management have been an important factor for many good reasons such as giving on time rescue services, in case of GPS; in none availability of satellite plane of view directing users to their destination and many other useful applications. Location-sensing or localization is an automatic means of position determination for the user through signal detection from their devices. Efficient location-sensing require sampling of fewer data blocks from received signal and in many cases continuous signal is not received, fewer or interrupted signal has been detected. From these few samples based on sparsity technique location estimation is performed.

This paper discusses different present techniques for localization of user through compressed sensing. Localization has gained its popularity in many domains including mobile ad-hoc and vehicular networks, robotics and Public Protection and Disaster Relief (PPDR) communication system. There are surveys [1][37] purely based on location-sensing techniques through trilateration methods, none at the moment were related to compressivesensing for localization of user nodes.

The outline of this survey is as follows: Section 2 discusses the main challenges and parameters for accurate localization of the user. Also what were the drawbacks of non-compressive techniques previously used for location- sensing. Later in the section 3, compressive sensing is explained and the re-formulation of the location parameters in form of sparse values is explained. In section 4 focus will be on the effective algorithms for localization explaining the sparse techniques and recent developments to perform efficient localizations.

\section{Localization Issues and Parameters}

\subsection{Linearizing Vs Non- Linear}

For location estimation usually parameters are taken into 2 or 3D dimensional coordinates. In cellular network location parameters are taken within the network without the aid from external resources such as GPS. Mostly UE is known in normal days and if not known the parameters are detected from within the network. Not like GPS, cellular network localization parameters are detected from limited region of interest (ROI). While detecting signals the nodes may be moving generating time-stamped measurements. These parameters may be in non-linear coordinates. These parameters should be combined to form a trajectory leading to the user location.

For accurate and sparse calculations (discussed in next section 3) all parameters are converted to linear parameters. Geometric methods can locate an object distance and measurements. Before sparsity was not introduced multiple dimensional coordinates were used. Following kinds of non- 
linear parameters exists

1) Lateration-Single Dimension

2) Trilateration-2D

3) Multi-lateration-3D

Every UE exhibits three or more parameters. For deploying compressive sensing, an efficient and less complex computation requires to convert all parameters in lateration to mere approximation values. The description is illustrated in the following figure 1 .
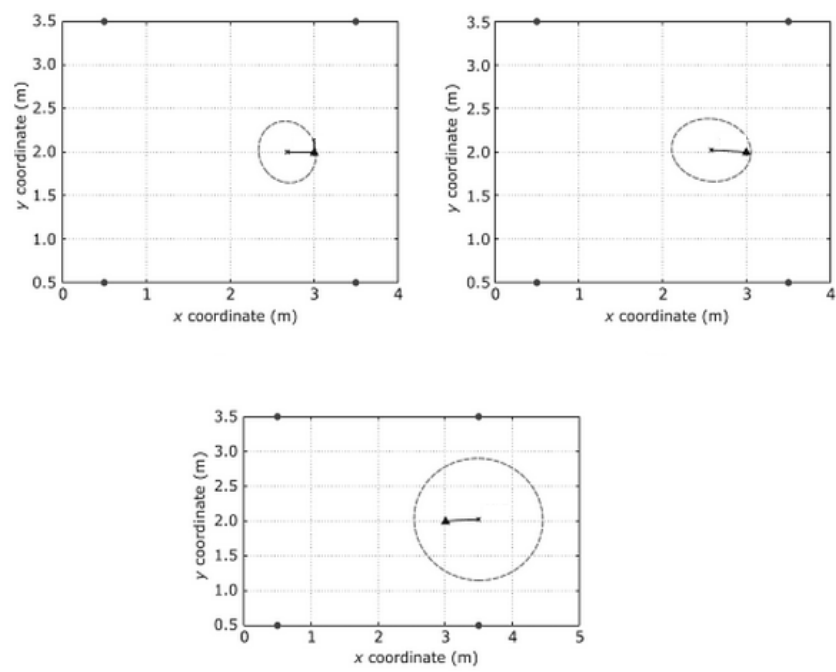

Figure 1. Location-sensing coordinates measurement

Location estimate in cellular/wireless networks are mostly processed through "RF finger prints" terminology. It is very similar to human finger printing. UE location-dependent signal parameters are extracted accordingly with their timeaverages. There are two types of RF finger printing either reference or target [2]. The parameters obtained are unique set of geographic coordinates. A simple set of data matrix is shown below.

$$
A=\left[\begin{array}{lcc}
I D_{1} & \mathrm{RSS}_{1} & \mathrm{RTD}_{1} \\
\ldots & \ldots . . & \cdots . . \\
\mathrm{ID}_{\mathrm{n}-1} & \mathrm{RSS}_{\mathrm{n}-1} & \mathrm{RTD}_{\mathrm{n}-1}
\end{array}\right]
$$

By using number of mathematical techniques such as Euclidean distance or Sum of Absolute Difference (SAD) these three dimensional values compute the distances with reference with the adjacent or known coordinate as shown in following equation

$$
d_{i, j}={\sqrt{\sum_{m=1}^{N}\left(\left\lfloor\frac{S_{i, j}{ }^{\prime}\left(n_{m}, 2\right)-A(m, 2)}{\delta}\right\rfloor\right)^{2}}}^{2}
$$

Here in above equation $S_{i, j}$ is the N-dimensional RSS space. As discussed above for localization many non-linear parameters were considered and computed. As the paper concentrate on compressive sensing effectiveness for localization rather than using trilateral coordinate system, further in next section sparsity in compressive sensing methodology is discussed in details.

\section{Fundamentals of Compressive Sensing}

\subsection{Sparse Representation}

Taking the location parameters from region of interest (ROI) and re-formulating it in $l$-minimization matrix for compressive sensing on the data is termed as sparse representation. The reason to apply sparse transformation for location estimation is due to the in-efficiency of locationsensing technology those require computation on large amount of data that cost overhead to its management and require high budget for hardware and software. Reformulation in compressive sensing provides fundamentally advance approach for cost-effective and time-consuming solutions.

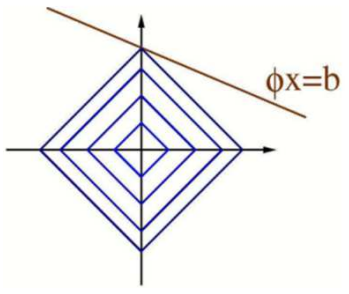

Figure 2. L-minimization [3]

By using fewer samples in linear domain compressive sensing implies sparsity. The explanation can be put forwarded as having a unknown signal vector $£^{\mathrm{N}}$, it is sampled using $\mathrm{n}$ functions for linearizing and later reconstruct it, where $\mathrm{n}<\mathrm{N}$ when signal space is bigger than measurements. Nearly from mid-eighteen it has been researched that minimization on 1-norm can recover sparse measurements as illustrated in above figure. Usually a sparse matrix derived from discrete-time domain signal is represented as follow

$$
\begin{gathered}
\Theta=\left\{\Omega_{i}\right\}_{i=1}^{M} \\
\Psi=\Theta e
\end{gathered}
$$

The discrete signal is represented as $\mathrm{S}^{\mathrm{M}}$ and e is $\mathrm{M} \times 1$ column vector of weighted product of co-efficient

$$
e_{i}=\left\langle\Psi, \Omega_{i}\right\rangle=\Omega_{i}^{T} \Psi
$$

The " $T$ " symbol denotes transpose of the sparse vector. The above equation is the sparse representation of signal. Only the basis linear combination of $k$ vectors are considered, meaning their values are the most significant such as

$$
\begin{aligned}
& \text { if } e_{i}=>k \neq 0 \\
& \text { then } M-k=0 \quad(k<M) \\
& M=(k \log N / k)
\end{aligned}
$$

Where $e_{i}$ is the linear projection of $M$ signal, having $N$ as intermediate acquiring samples. In a matrix if most of the 
elements are non-zero then the matrix or vector is considered dense not sparse matrix. Transform coding is successfully processed on the data samples those are k-sparse signals through compressive sensing. This framework is considered incoherent and represented as sparse representation. There exists number of different techniques for sparsing the data such as wavelet transformation, Logan phenomena, Lasso, the matching pursuit and least absolute shrinkage. Using not all signal samples but only few intervals is actually sparsity of signal where the sample is most weighted one.

\subsection{Compressive Sensing vs Data Compression}

There are two types of compression lossless and lossy. Compression sensing and data compression are two very different technologies. Before discussing compressive sensing in detail, the difference between two techniques should be well cleared. Data compression is a methodology of discarding and reducing data for increasing bit storage. There are number of different models and coding techniques for performing data compression.

Compressive sensing (CS) is very similar to transform coding, involving large amount of data. Transforming code process input signals into dense form of high dimensional space. The signal is sampled into sparsity form in a known transform domain. By sparsity it is meant, the matrix having samples of most weighted coefficients of a received signal that through transformation becomes zero.

\subsection{Spatial Sparsity in ROI}

Incoherency and sparsity are the two main pillars on which CS relies. High-dimensional signals especially trilateral coordinates for localization can easily be presented using few small set of variables and co-efficient through sparsity as shown in the figure 3.

\section{Present Techniques}

This section discusses present algorithms and methodologies for localization of user nodes through compressive sensing. As discussed in above sections the significance of sparsity theory over certain old techniques like FFT and Nyquist sampling theorem. In the following algorithm [5], a pair-wise distance measured matrix is derived by using sparsity. The central node only transmit small noisy compressive signal and a pair-wise matrix is constructed from those samples. CS uses 1-minimization matrix to find pair-wise matrix through sparsing. By applying 1-minimization algorithm, a sparse pair-wise distance matrix is reconstructed for learning locations of nodes. Suppose $S_{k} \in R O I^{n}$ is a sparse matrix $S$ having pair-wise distance values. In the matrix each value is a two dimensional location vector as expressed in equation 4.1 .

$$
\begin{gathered}
S_{k}=\left[S_{k 1}, S_{k 2}, \ldots \ldots \ldots S_{k n}\right] \\
\left\|S_{k i}\right\|_{0} \leq k, i \in\{1,2, \ldots . ., n\}
\end{gathered}
$$

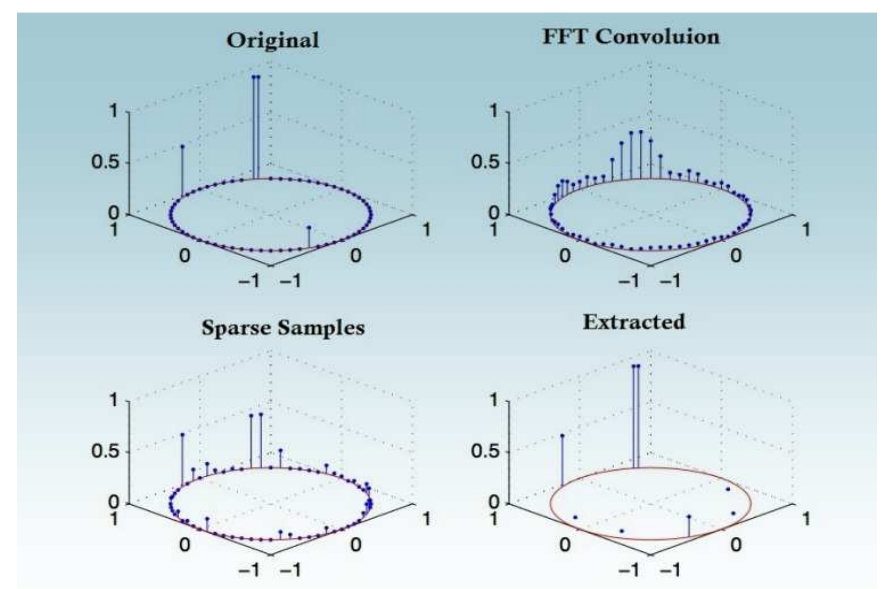

Figure 3. Sparse Data Samples

Further on three steps are performed on the matrix, in step 1, Floyd or Dijkstra path algorithm is applied on the values to recover sparse pair-wise values. In step 2, MDS algorithm is implied on the resultant matrix S'. The output from step 1 and 2 gives 3D relative coordinates of the nodes. Since this technique derive $3 \mathrm{D}$ coordinates for single node, the next techniques uses compressive sensing to derive the location of multiple points. The next popular technique [8] was evolved for missile launch system not for PPDR or public service schemes. The algorithm is very simple and straight forward by approaching the problem through Received Signal Strength (RSS) parameters. The RSS values are stored in a sparse matrix for pin-pointing the multiple location targets. The locations are then extracted from the sparse values through 1-minimization matrix technique. Like previously discussed techniques that measured the k-sparse representations, instead RSS measurements in Mdimensional coordinates are measured accurately by convoluting with original received signal according to below equation

$$
b=\Omega \Theta \xi+\varepsilon
$$

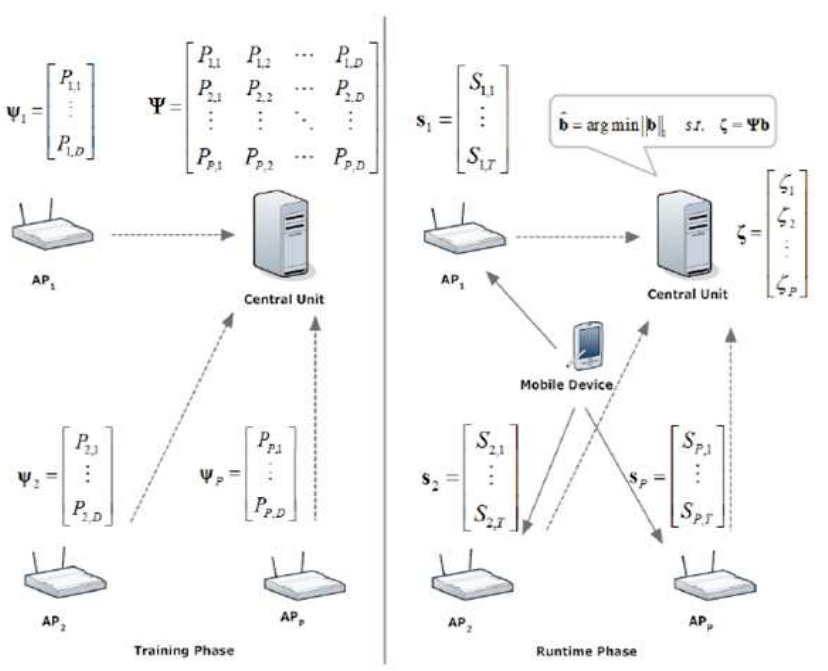

Figure 4. Coordinate Matrix [8] 
Where $\Omega$ is the sparse matrix having sparse coefficients $\mathcal{E}$

A fixed power definition is specified through adopted channel model and according to the RSS matrix readings on the grid scale the location of the targets are estimated. The following algorithm [10] emphasize main concern on the distribution of the estimation for low-dimensional location coordinates. A projection matrix is specified that is incoherent with the sparse matrix. This algorithm is based on the spatial sparsity representation. If the received signal having of $l$ length then $l+2$ parameters would be required for the estimation of location points on the $\mathrm{M}$ target locations having $\mathrm{k}$ sparse samples containing amplitudes of source signals referred as "Localization via spatial sparsity". Algorithms [7][9], [10] is based on both previous techniques [4][5] discussed above. Extracting the received signal strength and plotting over $\mathrm{k}$ sparse spree, a simple illustration is shown in figure 4.

A new technique is proposed in algorithm [12] defined as Greedy Matching Pursuit "GMP". GMP is an algorithm similar to OMP and CoSAMP [39] algorithms that could offer much better performance in regard to the unknown target locations from a measured signal. By adopting target energy decay model [40], [41] the states of signal energy received at certain location for pointed target from another location $\mathrm{j}$ is approximated as:

$$
C_{i j}=\frac{J_{0} G_{i j}}{d_{i j}^{\alpha}}
$$

Here $J_{0}$ is the received signal intensity at $\mathrm{i}, d_{i j}$ is the derived distance from Euclidean formula between the know target location $\mathrm{i}$ with the required / estimated target location $\mathrm{j}$, $G_{i j}$ holds the Raleigh fading for the received target signal. Sparsity is implied on the resultant matrix, after getting sparse representation points on the grid, energy of the target signal will be highest where there are most of the targets resides.

\section{Acknowledgements}

I would like to acknowledge TRENDS Lab of ITU University, Lahore Pakistan for their support and guidance in writing in this paper. Also I am thankful for ITU University administration to give access to their library resources for further literature review and allow me to use the material in research work.

\section{References}

[1] J. F. Jiang, G. J. Han, C. Zhu, Y. H. Dong, N. Zhang, "Secure localization in wireless sensor networks: A survey", Journal of Communications, vol.6, no.6, pp.460-470, 2011.

[2] Reza Zekavat, R. Michael Buehrer, "Handbook of Position Location: Theory, Practice and Advances," ISBN: 978-0-47094342-7
[3] Olga V.Holtz, "Compressive sensing: a paradigm shift in signal processing", Dec, 2008

[4] Richard G. Baraniuk, "More Is Less: Signal Processing and the Data Deluge",DOI: 10.1126/science.1197448 , 717 (2011); 331 Science

[5] Chen Feng, Shahrokh Valaee1, Zhenhui Tan Department of Electrical and Computer Engineering, University of Toronto, State Key Laboratory of Rail Traffic Control and Safety, Beijing Jiaotong University, "Localization of wireless sensors using compressive sensing for manifold learning," IEEE The 20th Personal, Indoor and Mobile Radio Communications Symposium, PIMRC, 2009.

[6] C. Feng, S. Valaee, and Z. Tan, "Multiple target localization using compressive sensing," in GLOBECOM'09: Proceedings of the 28th IEEE conference on Global telecommunications, 2009, pp. 4356-4361.

[7] J. geun Park, E. D. Demaine, and S. Teller, "Moving-baseline localization," in Proceedings of Information Processing in Sensor Networks (IPSN), 2008, pp. 15-26.

[8] S. Nikitaki and P. Tsakalides, ldquo, "Localization in Wireless Networks via Spatial Sparsity," Proc. Conf. Record of the 44th Asilomar Conf. Signals, Systems and Computers (ASILOMAR ',10), pp. 236-239, Nov. 2010.

[9] C. R. Berger, Z. Wang, J. Huang and S. Zhou "Application of compressive sensing to sparse channel estimation", IEEE Commun. Mag., vol. 48, no. 11, pp.164 -174 2010

[10] V. Cevher, M. F. Duarte and R. G. Baraniuk "Distributed target localization via spatial sparsity", 16th Eur. Signal Process. Conf., 2008.

[11] S. Nikitaki and P. Tsakalides, "Localization in wireless networks based on jointly compressed sensing," Proc. of European Signal Proc. Conf. (EUSIPCO), pp. 1809 - 1813, Aug.-Sept. 2011.

[12] B. Zhang, X. Cheng, N. Zhang, Y. Cui, Y. Li, and Q. Liang, "Sparse target counting and localization in sensor networks based on compressive sensing," in Proc. IEEE INFOCOM, pp. 2255-2263, 2011.

[13] Wael Guibène and Dirk Slock, "Cooperative Spectrum Sensing and Localization in Cognitive Radio Systems Using Compressed Sensing" Hindawi Publishing Corporation, Journal of Sensors, Volume 2013, Article ID 606413, 9 pages, http://dx.doi.org/10.1155/2013/606413

[14] W. Guibene and D. Slock, "A combined spectrum sensing and terminals localization technique for cognitive radio networks," in Proceedings of the IEEE 8th International Conference on Wireless and Mobile Computing, Networking and Comm's (WiMob '12), 2012.

[15] Sofia Nikitaki University of Crete \& FORTH, Heraklion, Greece, Panagiotis Tsakalides University of Crete \& FORTH, Heraklion, Greece, "Decentralized indoor wireless localization using compressed sensing of signal-strength fingerprints", PM2HW2N '12, Pages 37-44, ACM New York, NY, USA C2012, ISBN: 978-1-4503-1626-2 doi $>10.1145 / 2387191.2387198$

[16] Gan, Ming; Guo, Dongning; Dai, Xuchu, "Distributed Ranging and Localization for Wireless Networks via Compressed Sensing", eprint arXiv:1308.3548, Publication Date: $08 / 2013$ 
[17] Lanchao Liu, Zhu Han, Zhiqiang Wu, Lijun Qian, "Spectrum Sensing and Primary User Localization in Cognitive Radio Networks via Sparsity" , EAI Endorsed Transactions on Wireless Spectrum, Copyright (C) 2014, doi:10.4108/ws.1.1.e2

[18] R. M. Vaghefi and R. M. Buehrer, "Improving positioning in LTE through collaboration," in Proc. IEEE WPNC, 2014.

[19] Raja Jurdak, X. Rosalind Wang, Oliver Obst, and Philip Valencia, CSIRO ICT Centre, Australia, "Wireless Sensor Network Anomalies: Diagnosis, and Detection Strategies", A. Tolk and L.C. Jain (Eds.): Intelligence-Based Systems Engineering, ISRL 10, pp. 309-325.

[20] Sheenam, Navdeep Kaur, SBSTC, Ferozepur, India, "Improvement of Energy Efficiency of Compressive Sensing in Wireless Sensor Networks", ISSN 2348-5426 International Journal of Advances in Science and Technology (IJAST) Vol 2 Issue 2 (June 2014)

[21] W. Guibene and D. Slock, "Cooperative spectrum sensing and localization in cognitive radio systems using compressed sensing," Journal of Sensors, vol. 2013, Article ID 606413, 9 pages, 2013.

[22] K. Hayashi, M. Nagahara, and T. Tanaka, "A user's guide to compressed sensing for communications systems, " IEICE Trans. on Communications, vol. E96-B, no. 3, pp. 685-712, Mar. 2013.

[23] Joseph Lardies, Hua MA, Marc Berthillier. Source localization using a sparse representation of sensor measurements. Soci'et'e Fran, caise d'Acoustique. Acoustics 2012, Apr 2012, Nantes, France. $<$ hal-00810912>

[24] D.L Donoho and B. Logan, "Signal recovery and the large sieve,” SIAM J. Appl. Math., vol.52, no.2, pp.577-591, April 1992

[25] S.G. Mallat, "A Wavelet Tour of Signal Processing", Third ed. The Sparse Way, Academic Press, 2008.

[26] P.Buhlmann and S. van de Geer, Statistics for HighDimensional Data: Methods, Theory and Applications, Springer, 2011.

[27] S.G. Mallat and Z.Zhang, "Matching pursuits with timefrequency dictionaries," IEEE Trans. Signal Process., vol.41, no.12, pp.3397-3415, Dec. 1993

[28] R. Tibshirani, "Regression shrinkage and selection via the lasso,” J.R. Statist. Soc. B, vol.58, no.1, pp.267-288, 1996

[29] J.L. Starck, F.Murtagh, and J.M. Fadili, Sparse Image and Signal Processing: Wavelets, Curvelets, Morphological Diversity, Cambridge University Press, 2010.

[30] J. Yoo, C. Turnes, E. Nakamura, C. Le, S. Becker, E. Sovero, M. Wakin, M. Grant, J. Romberg, A. Emami-Neyestanak, and E. Cand'es, "A compressed sensing parameter extraction platform for radar pulse signal acquisition," Submitted to IEEE J. Emerg. Sel. Topics Circuits Syst., February 2012.

[31] W. Dai, O. Milenkovic, Subspace pursuit for compressive sensing: Closing the gap between performance and complexity, available at: http://www.dsp.ece.rice.edu/cs/SubspacePursuit.pdf (preprint)

[32] I. F. Gorodnitsky and B. D. Rao, "Sparse signal reconstruction from limited data using FOCUSS: A re-weighted minimum norm algorithm," IEEE Transactions on Signal Processing, vol 45, no. 3, pp. 600-616, 1997.

[33] V. Cevher, A. C. Gurbuz, J. H. McClellan, and R. Chellappa, "Compressive wireless arrays for bearing estimation," in IEEE Int. Conf. on Acoustics, Speech and Signal Processing (ICASSP), Las Vegas, NV, Apr. 2008.

[34] D. Malioutov, M. Cetin, and A. S. Willsky, "A sparse signal reconstruction perspective for source localization with sensor arrays," IEEE Transactions on Signal Processing, vol. 53, no. 8, pp. 3010-3022, 2005.

[35] D.Model and M. Zibulevsky, "Signal reconstruction in sensor arrays using sparse representations," Signal Processing, vol. 86, no. 3, pp. 624-638, 2006.

[36] A. C. Gurbuz, V. Cevher, and J. H.McClellan, "A compressive beamformer," in IEEE Int. Conf. on Acoustics, Speech and Signal Processing (ICASSP), Las Vegas, NV, 2008.

[37] Isaac Amundson and Xenofon D. Koutsoukos, "A Survey on Localization for Mobile Wireless Sensor Networks", R. Fuller and X.D. Koutsoukos (Eds.): MELT 2009, LNCS 5801, 2009, Pages: 235-254

[38] Guevara, J.; Jiménez, A.R.; Prieto, J.C.; Seco, F. Error Estimation for the Linearized Auto-Localization Algorithm. Sensors 2012, 12, 2561-2581.

[39] S. Foucart and H. Rauhut, A Mathematical Introduction to Compressive Sensing, Applied and Numerical Harmonic Analysis, DOI 10.1007/978-0-8176-4948-7 2, (C) Springer Science+Business Media New York 2013

[40] M. Ding, F. Liu, A. Thaeler, D. Chen, and X. Cheng, "Faulttolerant target localization in sensor networks," in EURASIP J. Wirel. Commun. Netw., vol. 2007, no. 1, 2007, pp. 19-28.

[41] T. Clouqueur, K. K. Saluja, and P. Ramanathan, "Fault tolerance in collaborative sensor networks for target detection," in IEEE Transactions on Computer, vol. 53, no. 3, 2004, pp. $320-333$.

[42] Marco F. Duarte, "Localization and Bearing Estimation via Structured Sparsity Models," IEEE Statistical Signal Processing Workshop (SSP), 2012, Ann Arbor, MI, pp. 333336.

[43] Emamnuel J. Candès, "Compressive sampling", Applied and Computational Mathematics, California Institute of Technology, Pasadena, CA 91125, U.S.A

[44] Lei Liu Jin-Song Chong, Xiao-Qing Wang, and Wen Hong, "Adaptive Source Location Estimation Based on Compressed Sensing in Wireless Sensor Networks" International Journal of Distributed Sensor Networks, Volume 2012 (2012), Article ID 592471, 15 pages, http://dx.doi.org/10.1155/2012/592471 